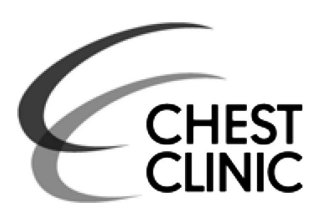

published online only. To view please visit the journal online (http://dx.doi.org/10.1136/ thoraxjnl-2014-205720).

${ }^{1}$ Department of Medicine, Division of Medical Oncology, Duke University Medical Center, Durham, North Carolina, USA

${ }^{2}$ Department of Clinical Sciences, Division of Respiratory Medicine \& Allergology, Lund University, Lund, Sweden

${ }^{3}$ Discipline, Palliative and Supportive Services, Flinders University, Adelaide, South Australia, Australia

${ }^{4}$ Health Services Research and Development, Durham Veteran's Affairs Medical Center, Durham, North Carolina, USA

${ }^{5}$ Department of Medicine, Division of General Internal Medicine, Duke University Medical Center, Durham, North Carolina, USA

${ }^{6}$ Department of Biostatistics and Bioinformatics, Duke University, Durham, North Carolina, USA

\section{Correspondence to} Dr Amy P Abernethy, Duke University Medical Center, Box 3436, Durham, NC 27710 USA; amy.abernethy@duke.edu

Received 12 May 2014 Revised 15 October 2014 Accepted 14 November 2014 Published Online First

3 December 2014

\title{
Oxygen for relief of dyspnoea in people with chronic obstructive pulmonary disease who would not qualify for home oxygen: a systematic review and meta-analysis
}

\author{
Hope E Uronis, ${ }^{1}$ Magnus P Ekström, ${ }^{2,3}$ David C Currow, ${ }^{3}$ Douglas C McCrory, ${ }^{4,5}$ \\ Gregory P Samsa, ${ }^{6}$ Amy P Abernethy ${ }^{1,3}$
}

\section{ABSTRACT}

We searched MEDLINE, EMBASE and the Cochrane Controlled Trials Register to determine whether oxygen relieves dyspnoea in mildly or non-hypoxemic COPD and included 18 randomised controlled trials (431 participants) in the meta-analysis using Cochrane methodology. Oxygen therapy reduced dyspnoea when compared with medical air; standardised mean difference $-0.37\left(95 \% \mathrm{Cl}-0.50\right.$ to $\left.-0.24 ; \mathrm{I}^{2}=14 \%\right)$. In a priori subgroup and sensitivity analyses, dyspnoea was reduced by continuous oxygen during exertion but not short-burst oxygen therapy. Continuous exertional oxygen can relieve dyspnoea in mildly or non-hypoxemic COPD, but evidence from larger clinical trials is needed.

\section{INTRODUCTION}

Dyspnoea is a common and distressing symptom for patients with COPD. The management of dyspnoea includes optimised COPD treatment with bronchodilators, pulmonary rehabilitation, regular use of low-dose opioids and home oxygen therapy.

It is unknown whether oxygen relieves dyspnoea in patients with mild or non-hypoxemic COPD (palliative oxygen). Home oxygen therapy does improve survival in COPD with severe hypoxaemia $\left(\mathrm{P}_{\mathrm{a}} \mathrm{O}_{2} \leq 7.3 \mathrm{kPa}\right)$ or mild hypoxaemia $\left(\mathrm{P}_{\mathrm{a}} \mathrm{O}_{2}\right.$ $7.4-7.9 \mathrm{kPa}$ ) in the setting of pulmonary hypertension, cor pulmonale and/or secondary polycythaemia (haematocrit $>55 \%$ ). ${ }^{1}$ Effects of oxygen on symptoms, including dyspnoea, in mild to moderate hypoxaemia are unknown.

Despite sparse supporting evidence, palliative oxygen is frequently prescribed to improve dyspnoea. Oxygen therapy is, however, costly and may be ineffective or might cause complications including nasal irritation, burns secondary to smoking, hyperoxia-related vasoconstriction and exacerbation of hypercapnia. Data on the efficacy of palliative oxygen therapy are needed.

We therefore conducted a systematic review and meta-analysis to determine whether oxygen therapy provides symptomatic relief in COPD patients with breathlessness who do not qualify currently for long-term oxygen.

\section{METHODS AND MATERIALS}

This paper summarises the full 2011 review published by the Cochrane Library. ${ }^{2}$

\section{Search strategy}

We searched MEDLINE (1966 to November 2009), EMBASE (1980 to November 2009) and the Cochrane Airways Group Specialised Register (through November 2009) as detailed in the online supplement. Additional manuscripts were identified by searching references lists of articles and published reviews that met the inclusion criteria.

\section{Study selection}

Inclusion criteria were: (1) randomised controlled trial; (2) participants were adults with COPD; (3) mean $\mathrm{PaO} 2 \geq 7.3 \mathrm{kPa}$; (4) subjects were not already receiving home oxygen therapy; (5) oxygen versus medical air as intervention; (6) intervention delivered through non-invasive method (nasal cannula, mouthpiece or mask); and (7) dyspnoea as one study outcome. Two investigators (HEU, APA) screened abstracts independently. Differences in judgment were resolved by consensus conference. The flow of articles reviewed is presented in the online supplementary figure S1.

\section{Data extraction}

Basic study parameters were abstracted (by HEU and verified by APA) into evidence tables. When necessary, the authors of the primary studies were contacted to obtain additional information.

\section{Types of oxygen therapy}

The oxygen therapy was categorised as either shortburst when given for a short period pre-exertion or postexertion, or as continuous (during exertion and/ or at rest) including domiciliary oxygen therapy.

\section{Quality assessment}

Each study was assessed for internal validity (randomisation, blinding and description of withdrawals/dropouts) and external validity (descriptions of subjects and interventions, and adequately reported dyspnoea outcomes) using the Jadad score (5-point scale; higher score indicating better validity), shown in online supplementary table S1.

\section{Data analysis}

The primary outcome of interest was the effect on patient-rated dyspnoea. Secondary outcomes were effect on quality of life (QOL) and patient treatment preference. Crossover comparisons were analysed, 
when available, using paired tests. Correlations and SEs were calculated as described elsewhere. ${ }^{2}$ Effect estimates measured on different dyspnoea scales were standardised by dividing each mean difference with its SD, giving the standardised mean differences (SMDs). Effect sizes were calculated for random-effects models using RevMan 5 (Cochrane Informatics and Knowledge Management, Oxford, UK) and reported as SMD with 95\% CIs. Heterogeneity was described as the $\mathrm{I}^{2}$ for each measure and explored through the following prespecified subgroup and sensitivity analyses: studies with mean $\mathrm{PaO}_{2}<9.3$ compared with $\geq 9.3 \mathrm{kPa}$; studies of short-burst oxygen compared with other studies; and excluding studies using imputed data.

\section{RESULTS}

\section{Excluded studies}

Ten studies were not included in the meta-analysis due to insufficient data (seven crossover trials), significant order effect (one crossover trial) and lack of crossover comparisons (two parallel group studies). Five of the excluded studies reported that oxygen improved dyspnoea while four reported no significant effect.

\section{Study characteristics}

In total, 28 studies, comprising 31 study comparisons and 702 participants met the inclusion criteria (see online supplementary table S1). Of the 28 included studies, 23 were blinded, randomised, crossover trials and five were randomised, controlled, parallel arm trials.

Most studies required patients to have moderate to severe COPD for study entry. All studies compared oxygen with medical air; 23 studies (82\%) provided continuous oxygen during exertion, four studies (14\%) provided short-burst oxygen and one study provided oxygen at rest.

Study details are reported in online supplementary table S1. Methods were poorly reported in most of the included studies. While all studies were described as randomised, we could verify that sequence generation was adequate in only six studies. Masking of treatment was undertaken in a number of studies, but we were unable to determine how blinding had been achieved in five studies.

\section{Treatment efficacy}

Primary outcome: dyspnoea

This analysis included 18 studies (427 patients; 21 study comparisons) (figure 1). Oxygen relieved dyspnoea in mildly- or non-hypoxemic COPD patients (SMD $-0.37,95 \% \mathrm{CI}-0.50$ to $-0.24)$ more effectively than medical air. Significant heterogeneity was not observed $\left(\mathrm{I}^{2}=14 \%, \mathrm{p}=0.24\right)$.

\section{A priori subgroup and sensitivity analyses}

Oxygen relieved dyspnoea both in studies with a mean $\mathrm{P}_{\mathrm{a}} \mathrm{O}_{2}$ $\geq 9.3 \mathrm{kPa}$ (12 studies; SMD $-0.42,95 \%$ CI -0.61 to -0.24 ; $\left.\mathrm{I}^{2}=28 \%\right)$ and in studies with a mean $\mathrm{P}_{\mathrm{a}} \mathrm{O}_{2}<9.3 \mathrm{kPa}$ (six studies; SMD $-0.25,95 \% \mathrm{CI}-0.50$ to $-0.00 ; \mathrm{I}^{2}=14.2 \%$ ), as shown in online supplementary figure S2.

The four studies of short-burst oxygen showed that the method did not relieve dyspnoea (SMD 0.01 , 95\% CI -0.26 to $\left.0.28 ; \mathrm{I}^{2}=0 \%\right)$, whereas 14 studies of continuous oxygen during exertion improved dyspnoea (SMD $-0.46,95 \%$ CI -0.59 to $-0.33 ; \mathrm{I}^{2}=0.0 \%$ ) (see online supplementary figure S3).

Sensitivity analysis, using six studies for which SMD and variance, was obtained without any data imputation and showed a stable beneficial effect of oxygen on dyspnoea (SMD -0.36 , $95 \% \mathrm{CI}-0.64$ to $-0.09 ; \mathrm{I}^{2}=59 \%$ ).

\section{Secondary outcomes: QOL and patient preference}

Meta-analyses of secondary outcomes could not be performed due to heterogeneity in data presentation and outcome measurements.

QOL was reported in three of 28 included studies. One study found no statistically significant differences in the Chronic Respiratory Questionnaire (CRQ), Short Form 36 (SF-36), or the Hospital Anxiety and Depression Scale (HADS). The second study reported statistically significant improvements in all domains of the CRQ (fatigue, $p=0.02$; emotional function, $\mathrm{p}=0.006$; mastery, $\mathrm{p}=0.008$; total, $\mathrm{p}=0.002)$, both domains of the HADS (anxiety, $p=0.009$; depression, $p=0.05$ ) and in several domains of the SF-36. The third study found no statistically significant difference in the CRQ.

Blinded participant preferences were reported in three studies. In the first study, $41 \%$ of participants thought to be benefiting from oxygen did not wish to continue to receive

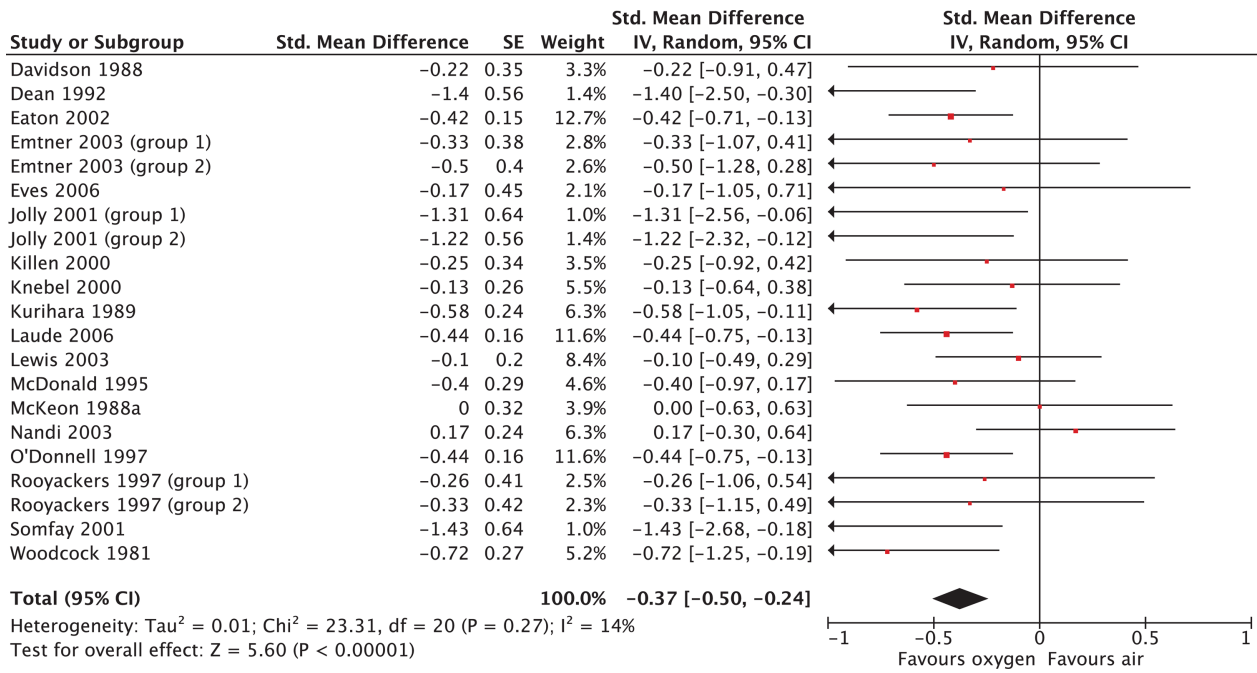

Figure 1 Forest plot summarising randomised trials of the symptomatic effect of oxygen on breathlessness in people with COPD who do not qualify for long-term oxygen therapy; all trials. 
therapy, $76 \%$ citing poor tolerability as the reason. In the second study, 12 of 18 subjects preferred oxygen, five before going up the stairs and seven after completing the ascent. Of the remaining six patients, three preferred room air before going up the stairs and three had no preference. In the third study, after 6 weeks home therapy with each gas, half of the patients preferred oxygen while the other half either preferred air or had no preference.

\section{DISCUSSION}

Continuous oxygen during exertion, but not short-burst therapy, reduced dyspnoea in mildly- and non-hypoxemic people with COPD who would not otherwise qualify for home oxygen therapy. The effect, SMD $-0.46(95 \% \mathrm{CI}-0.59$ to -0.33$)$ translates into a clinically meaningful reduction of about $1.2 \mathrm{~cm}$ on a $10 \mathrm{~cm} \mathrm{VAS.}^{3}$

Data on QOL and patient preference were sparse and conflicting making it impossible to perform a meta-analysis. The conflicting results may be due to differences or interactions of study design, patient population and small sample sizes. The burden imposed by home oxygen therapy may also outweigh any potential impact on QOL. Interestingly, 41\% of 'oxygen responders' in one study did not desire further therapy owing mainly to inconvenience or poor tolerability. QOL and other patient factors should be considered when deciding whether the benefits of palliative oxygen outweigh its inconveniences. Further research is needed.

Several limitations are noteworthy. Baseline dyspnoea, functional status or both were not presented in many studies, making it difficult to assess whether the participants were similar.

In conclusion, continuous oxygen during exertion relieved dyspnoea in people with COPD who would not otherwise qualify for home oxygen therapy due to mild or absent hypoxaemia. The effect might be mediated by reduced exertional hypoxaemia. The observed heterogeneity of study designs, however, makes it difficult to provide general recommendations. Two double-blind, randomised trials published after the completion of the review reported no relief of breathlessness from palliative oxygen when compared with medical air ${ }^{4}$ and no benefit from ambulatory oxygen. ${ }^{5}$ Decisions regarding prescription of oxygen for relief of dyspnoea in individuals not meeting criteria for long-term oxygen therapy should be made on an individual basis after careful consideration. Data from adequately powered clinical trials, including studies on cost-effectiveness, risk of possible hyperoxia-related adverse effects and fires, and future meta-analyses are needed to answer this question.

Acknowledgements We would like to thank the following individuals for their assistance with the initial database searches: Ms Anne Powers, Duke University Medical Center Library, Durham, North Carolina, and Ms Belinda Fazekas, Repatriation General Hospital, Daw Park, South Australia. We would also like to thank Ms Susan A Hansen and Ms Liz Arnold, Cochrane Airways Group, for their assistance with subsequent the final MEDLINE/EMBASE/CCTR searches and document acquisition. The authors also acknowledge Donald T Kirkendall, ELS, a Duke-employed medical editor, for his assistance in the preparation of this manuscript.

Contributors HEU, DCC, DCM, GPS and APA performed the study design and analysis. HEU, MPE, DCC, DCM, GPS and APA participated in the interpretation of the findings, writing and revision of the paper.

Funding This work was supported by the Agency for Healthcare Research and Quality (T32 HS000079) and National Institutes of Health (R01 AG026469-02).

Competing interests None.

Provenance and peer review Not commissioned; externally peer reviewed.

\section{REFERENCES}

1 Medical Research Council Working Party. Long term domiciliary oxygen therapy in chronic hypoxic cor pulmonale complicating chronic bronchitis and emphysema. Report of the Medical Research Council Working Party. Lancet 1981;1:681-6.

2 Uronis $\mathrm{H}, \mathrm{McC}$ rory DC, Samsa G, et al. Symptomatic oxygen for non-hypoxaemic chronic obstructive pulmonary disease. Cochrane Database Syst Rev 2011;(6): CD006429.

3 Johnson MJ, Bland JM, Oxberry SG, et al. Clinically important differences in the intensity of chronic refractory breathlessness. J Pain Symptom Manage 2013;46:957-63.

4 Abernethy AP, Mcdonald CF, Frith PA, et al. Effect of palliative oxygen versus room air in relief of breathlessness in patients with refractory dyspnoea: a double-blind, randomised controlled trial (NCT 00327873). Lancet 2010;376:784-93.

5 Moore RP, Berlowitz DJ, Denehy L, et al. A randomised trial of domiciliary, ambulatory oxygen in patients with COPD and dyspnoea but without resting hypoxaemia. Thorax 2011;66:32-7. 